\title{
Harness the power of endogenous neural stem cells by biomaterials to treat spinal cord injury
}

\author{
DE FILIPPIS Lidia ${ }^{1}$, SÜDHOF Thomas C. ${ }^{2,3^{*} \ddagger} \&$ PANG ZhiPing P. ${ }^{1}$ \\ ${ }^{1}$ Child Health Institute of New Jersey, Rutgers University Robert Wood Johnson Medical School, New Brunswick, NJ 08901, USA; \\ ${ }^{2}$ Howard Hughes Medical Institute Stanford University, Palo Alto, CA 94304, USA; \\ ${ }^{3}$ Department of Molecular and Cellular Physiology, Stanford University, Palo Alto, CA 94304, USA
}

Received October 8, 2015; accepted October 10, 2015; published online October 14, 2015

Citation: De Filippis L, Südhof TC, Pang ZPP. Harness the power of endogenous neural stem cells by biomaterials to treat spinal cord injury. Sci China Life Sci, 2015, 58: 1167-1168, doi: 10.1007/s11427-015-4943-Z

Spinal cord injury (SCI) is a devastating medical condition without a cure. Reestablishment of neuronal connections after spinal cord injury is the "holy grail" of SCI research. However, grafting exogenous cells, including neural stem cells and a variety of adult somatic cells, has had very limited success [1]. Two back-to-back papers published in the Proceedings of National Academy of Sciences USA by the $\mathrm{Li}$ and Sun groups have provided exciting information by bringing activated endogenous neurogenesis into play, using a biomaterial called chitosan that was loaded with neurotrophic factor 3 (NT3) to achieve slow release of the trophic factor. Moreover, these papers have provided a mechanistic insight using analyses of gene expression and neuronal function.

We now know that after SCI, the development of a non-permissive microenvironment by a combination of severance of axons and vascular structure, edema, infiltration of immune cells and progressive gliosis and scar formation creates a barrier for neuronal regeneration. It has been suggested that a neural stem/progenitor cells (NSCs) niche that resides in the central nervous system (CNS) can potentially be activated for neural regeneration, but little progress has been made in exploiting this process therapeutically in SCI.

In the first study by Yang et al. [2], the authors applied an innovative chitosan-NT3 biomaterial which not only

*Corresponding author (email: tcs1@stanford.edu)

$\ddagger$ SÜDHOF Thomas C., the winner of the 2013 Nobel Prize in Physiology or Medicine bridged the transected spinal cord, but also released NT3 slowly to provide a favorable microenvironment for NSC activation and migration. They transected and extracted a 5 mm spinal cord segment at T7/8, filled the gap immediately after the injury with NT3-chitosan, and followed up the recovery at different stages after the injury (from 30 days up to 1 year). The researchers checked tissue recovery in the lesion area as well as in regions that were rostral $(\mathrm{R})$ or caudal (C) to the lesion. In addition, using Basso, Beattie and Bresnahan (BBB) post injury locomotor analyses monitored functional recovery. The authors observed that BBB scores of animals in the NT3-chitosan group were much higher than those of the control groups up to 52 weeks (1 year) post injury, and that tissue repair was a slow process, with partial successful functional recovery dependent on the gradual release of NT3 by chitosan over time. To further test whether recovery of locomotion was due to the tissue regeneration, the authors resected the nerve terminals in the same lesion area and replaced the NT3-chitosan with a plastic diaphragm, which completely abolished restoration of locomotor behavior, and thus excluded the possibility that an intrinsic compensatory mechanism rescued the lesion. The anatomical and behavioral recovery was associated with functional synapse formation between the regenerated neurons within the chitosan conduit (the injury site). Next, the authors provided evidence that spontaneous activation of endogenous NSCs by injury and NT3 contributed to the generation of neurons. To further explore this hypothesis, 
the authors blocked NSC proliferation pharmacologically, and observed a $30 \%$ decrease in the amplitude of somatosensory evoked potentials (SEPs) and motor evoked potentials (MEPs) in the treated group, suggesting a consistent contribution of newly generated neurons to locomotor functional recovery. These experiments suggest that the establishment of a permissive microenvironment may be important for neuronal tissue regeneration.

In an accompanying paper by Duan et al. [3] from the same group, the authors used genomic analysis to probe the mechanism underlying the activation of endogenous NSCs, and to search for possible prohibitive factors that could hamper regeneration of neurons after SCI. Using an ingenuous analytical method called the Weighted Gene Coexpression Analysis (WGCNA), the authors found a gene expression pattern which suggested that the NT-3 chitosan bio-tube may dampen inflammatory immune processes [3], enhance formation of blood vessels, and promote de novo neurogenesis. WGCNA analysis involves identification of gene modules that are representative of pathological events, and potentially allows definition of diagnostic hallmarks and targets for future therapeutic interventions.

In recent years cell-based therapies have tried to utilize pluripotent stem cells and adult-derived stem cells of different origin or more mature cells such as mesenchymal stem cells, olfactory ensheathing cells, and Schwann cells [4]. Nonetheless, in the treatment of SCI, cell transplantation is often hampered by poor cell survival. To overcome this drawback, investigators have grafted cells in combination with biomaterials that are meant to physically mimic CNS tissue and to foster a permissive environment for cell survival, growth, and differentiation. However, the risk of immune rejection or tumor development remains a major drawback of this approach [5]. Therefore mobilization of an endogenous pool of NSCs may be a more promising approach, but currently no studies have produced a successful protocol to achieve this, and a variety of experimental data led to questions about the molecular mechanisms underlying functional recovery after SCI. One of the main reasons for this heterogeneity resides in the lack of a temporal analysis aimed at determining a pipeline of pathological hall- marks from SCI to recovery. The two recent papers addressed these critical hurdles in SCI treatment.

However, several critical questions still require clarification. For example, what are the cell types regenerated in the lesion site, what is their composition and, importantly, how do non-neuronal cells like astrocytes potentially contribute to the recovery [6]? Moreover, what is the relationship between the intact neural structure and the newly regenerated cells, including the synaptic connections of descending inputs, intraspinal circuits, or circuits formed within the new cells?

As a whole, the two studies by the $\mathrm{Li}$ and Sun groups report promising results suggesting that the slow release of a neurotrophin (NT3) by a biomaterial (chitosan) can be effective in promoting physiological repair processes in SCI and in dampening the deleterious effects secondary to the progression of chronic neuro-inflammation [2]. Moreover, using WGCNA, the authors established a gene- module analysis approach corresponding to various pathological events at different times after SCI, which provides mechanistic clues to potentially more effective treatments for SCI [3].

1 Plemel JR, Wee Yong V, Stirling DP. Immune modulatory therapies for spinal cord injury--past, present and future. Exp Neurol, 2014, 258: 91-104

2 Yang Z, Zhang A, Duan H, Zhang S, Hao P, Ye K, Sun Y, Li X. NT-3 chitosan elicits robust endogenous neurogenesis to enable functional recovery after spinal cord injury. Proc Natl Acad Sci USA, 2015, in press, 10.1073/pnas.1510194112

3 Duan H, Ge W, Zhang A, Xi Y, Chen Z, Luo D, Cheng Y, Fan K, Horvath S, Sofroniew MC, Cheng L, Yang Z, Sun Y, Li X. Transcriptome analyses reveal molecular mechanisms underlying functional recovery after spinal cord injury. Proc Natl Acad Sci USA, 2015, in press, doi: 10.1073/pnas.1510176112

4 Assuncao-Silva RC, Gomes ED, Sousa N, Silva NA, Salgado AJ. Hydrogels and cell based therapies in spinal cord injury regeneration. Stem Cells Int, 2015, 948040

5 Matsui T, Akamatsu W, Nakamura M, Okano H. Regeneration of the damaged central nervous system through reprogramming technology: basic concepts and potential application for cell replacement therapy. Exp Neurol, 2014, 260: 12-18

6 Falnikar A, Li K, Lepore AC. Therapeutically targeting astrocytes with stem and progenitor cell transplantation following traumatic spinal cord injury. Brain Res, 2015, 1619: 91-103

Open Access This article is distributed under the terms of the Creative Commons Attribution License which permits any use, distribution, and reproduction in any medium, provided the original author(s) and source are credited. 